\title{
Kissing Right? Absence of Rightward Directional Turning Bias During First Kiss Encounters Among Strangers
}

\author{
Jennifer R. Sedgewick ${ }^{1}$. Abby Holtslander ${ }^{1} \cdot$ Lorin J. Elias $^{1}$
}

Published online: 22 February 2019

(c) The Author(s) 2019

\begin{abstract}
When leaning toward a partner for a kiss, the direction that individuals turn their head when planting the kiss is found to vary based on the kiss's context; romantic kissing between adult couples is consistently directed rightward, though recently, a non-romantic kiss between parent-child couples was observed to be leftward. The current study further examines the lateral head-turning direction between non-romantic couples using a novel context: a first kiss between strangers. Observing strangers kissing was feasible due to a unique social media phenomenon; since 2014, 23 "First Kiss" online videos have emerged which depict kisses facilitated by the video's director between consenting strangers. The turning direction of 230 kissing couples were coded from the 23 First Kiss videos, and the proportion of right to left turns were almost equal; $51 \%$ of couples displayed a rightturn kiss, and $49 \%$ conveyed a left-turn kiss. Further, the proportion of right and left turns observed from our sample of strangers kissing were compared to Güntürkün's (in Nature 421:711, https://doi.org/10.1038/421711a, 2003) original study that examined authentic kissing between adult couples. A significantly different turning bias was exhibited. Because the kissing criterion was parallel between these studies, our study demonstrates that the context influenced the direction of bias, namely, that of a non-romantic kiss. We discuss the potential role of context and emotional lateralization on kissing laterality, and propose future directions to test these predictions.
\end{abstract}

Keywords Head-turning $\cdot$ Kissing $\cdot$ Context $\cdot$ Bias $\cdot$ Asymmetry $\cdot$ Laterality

\section{Introduction}

From kicking a ball to choosing which hand to write with, humans demonstrate various lateral preferences that are performed more frequently to the left or right. Motor asymmetries that are most often cited are the predominant right-sidedness for hand, foot, and eye dominance, all of which are posited to arise from innate mechanisms (Kumar et al. 2010; Mandal et al. 1992). Recently, an equally persistent right-side lateral preference has

Lorin J. Elias

Lorin.Elias@usask.ca

1 Department of Psychology, University of Saskatchewan, 9 Campus Drive, Saskatoon,

SK S7N 5A5, Canada 
been identified during head-turning behavior. Specifically, when facing towards a romantic partner and leaning in for a kiss, individuals tend to tilt their head to the right when planting the kiss more often than to the left.

Head-turning biases during kissing were discovered by Güntürkün (2003) by observing 124 lip-to-lip kisses between adult couples in public spaces (e.g., airport terminals, beaches, parks). From these observations, a disproportionate bias emerged; approximately $65 \%$ of couples turned to the right whereas $35 \%$ turned to the left. To eliminate the possibility that kisser A could have subtly communicated to kisser B the direction of turn for this nonverbal behavior, further studies utilized a nonhuman kissing partner: a human-sized mannequin head (Barrett et al. 2006; Ocklenburg and Güntürkün 2009; van der Kamp and Canal-Bruland 2011). When observing individual rather than joint-action turning direction, the head-turning bias persisted; 66\% (Shaki 2013) to 81\% (Barrett et al. 2006) of individuals from Western cultures exhibited a right-turned kiss.

Comparable to the theoretical mechanism guiding handedness, the right head-turning preference when kissing was speculated by Güntürkün (2003) to arise from an innate motor bias. This rationale was founded upon the observation that within the prenatal environment, fetuses develop a lateralized head position preference beginning from 38 weeks of gestation; specifically, the head tends to rest turned to the right as opposed to a mid-line position (Previc 1991; Ververs et al. 1994). After conception until approximately 3 months of age, a head position preference is also observed from infants' posture when oriented on their backs (i.e., their supine posture; Hopkins et al. 1987; Rönnqvist and Hopkins 1998). Head-turning asymmetries are thus described to be transient. They are one of our first observable motor preferences and disappear from typical developmental progress, but they are prominently displayed when leaning in for a kiss.

The congenital hypothesis has been the predominant theory for kissing laterality, though recent investigations challenge this theory. For instance, if the right-turn preference did arise from an innate mechanism, we would expect this directionality to persist across cultures akin to the right-side prevalence of handedness (Ardila et al. 1989; Dragovic 2004; Gilbert and Wysocki 1992). However, this is not the case. Shaki (2013) demonstrated that visuospatial habits guided by one's native reading direction influenced the directionality of bias. By conducting the study in geographic locations with official languages that read from left-to-right (Italian, English) and right-to-left (Hebrew, Arabic), a reversed bias was evident from both authentic kissing and on a mannequin-kissing task: left-to-right reading cultures displayed a right-turn bias, whereas right-to-left readers displayed a left-turn bias. Shaki (2013) also examined the correlation between turning preference and handedness, footedness, and eyedness, as these motor biases tend to exhibit a consistent directional pattern (Kumar et al. 2010; Mandal et al. 1992). Although a significant correlation was reported from Ocklenburg and Güntürkün (2009), the results from Shaki mirrored those from previous research by van der Kamp and Canal-Bruland (2011) and Barrett et al. (2006) which found no correlation between head-turning preference and innate motor biases.

If native reading direction were only a moderator of an innate head-turning bias, we should expect the right-side preference from left-to-right reading cultures to transcend kissing contexts, comparable to how individuals who use their right hand to write with a pen typically use the same hand to manipulate a paintbrush (Elias et al. 1998). Again, this is not the case. Sedgewick and Elias (2016) introduced a novel lip-kissing context: a kiss shared between a parent and child. Images of parent-parent kissing (romantic context) and parent-child kissing (parental context) couples were collected and coded for the direction of head-turn. Both contexts were considered to be emotional in their intent, but differed in 
their romantic motivation. The consistent right-turn bias was observed from kissers in the romantic context, though a left-turn bias was found for parent-child kissing partners. These findings demonstrate that contextual factors guided by the emotional intent of the kiss may play a role in the direction of lateral bias.

Although the assumption of kissing laterality originating from an innate mechanism is a logical prediction, the extant literature suggests that other factors contribute to the nonverbal communication of kissing. The purpose of the present study is to further explore the influence of kissing context, and specifically, between adult dyads. Although adults have several conventions of kissing, such as when greeting friends, within religious rituals, or when displaying affection, our context of interest is for a particularly novel one: a first kiss between strangers. The possibility of analyzing this obscure scenario was feasible due to a social media phenomenon stemming from one viral video.

In March 2014, New York clothing company Wren released a short film entitled First Kiss. Directed by Tatia Pllieva, First Kiss displays 20 unacquainted individuals who have consented to be randomly paired with each other to engage in a first kiss. The initiation of each kiss is captured, thus illustrating precisely the direction that individuals turn when approaching their partner. As one would imagine when kissing a stranger, the body language revealed is often awkward, though surprisingly for some couples, quite passionate (see Fig. 1 for illustrated examples). Prior to this study, 23 first kiss videos were available on the popular online video-sharing website, YouTube. Although we are trusting that these kisses are in fact between strangers and not actors, the opportunity to examine first kiss encounters has become a possibility because of this unique data driven by social media.

The First Kiss videos comprise the content of our analysis, to which the purpose of using this content is to examine the lateral turning direction of strangers engaging in a first kiss. This scenario is shared between adult couples, parallel to the kissing dyads from previous research, and kisses are qualified using comparable criteria: lip-contact, positioned face-to-face, and the absence of hand-held objects (Güntürkün 2003; Shaki 2013). Investigating strangers kissing is a departure from the previous studies in that it can simultaneously examine (1) a context whereby the motivation to deliver a quality kiss is likely decreased in comparison to kissing a romantically-desired partner, and (2) kisses are between individuals with no prior kissing habits established with each other. We presume a lack of emotional intention behind the kiss due to the artificiality of the scenario, as the
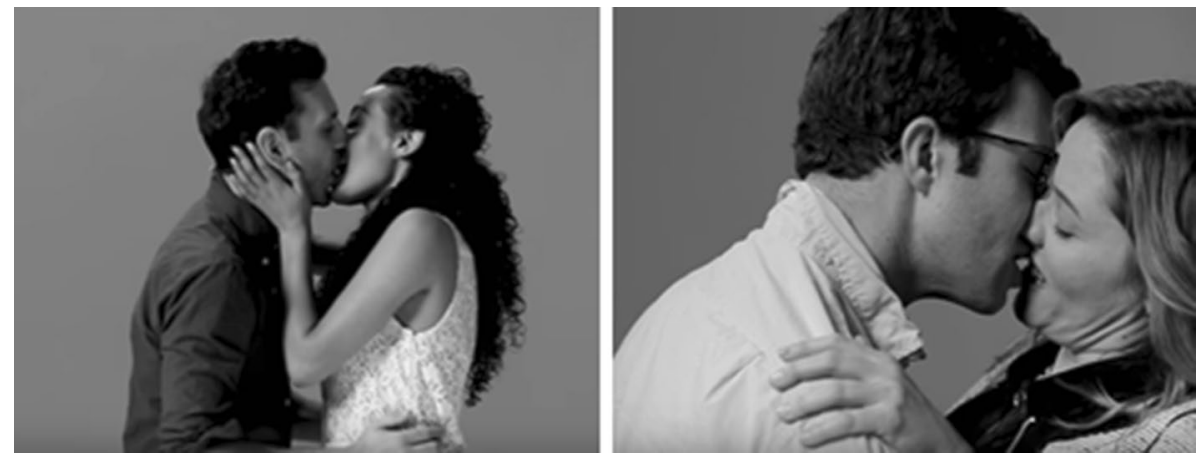

Fig. 1 Film stills from First Kiss. As conveyed by the body language, the images from left to right illustrate a passionate and awkward kissing interaction, respectively. Presented images were collected from the original First Kiss video: https://www.youtube.com/watch?v=IpbDHxCV29A 
couples are not only strangers but also do not get to voluntarily choose their kissing partner. Kisses from previous studies were assumed to be of a romantic type because they were described to be between adults, on the lips, and from regions where lip-kissing is not typically performed as a social gesture.

Based on the previous literature examining adult couples kissing, we predict that the head-turning direction of strangers kissing will be biased to the right significantly more often than the left. Further, we should expect a right-turn bias to emerge because the geographic location in which our participant data, the couples from the First Kiss videos, were filmed in cities with official languages that have a left-to-right orthography. A significant right-turn bias will provide theoretical support for the congenital account of kissing laterality by demonstrating that the direction of lateral bias persists across contexts, similar to other motor preferences. A disparate lateral preference such as the left-turn bias observed from child-parent kissing dyads (Sedgewick and Elias 2016), however, will provide further evidence against this prediction.

The extant literature that examines how motor asymmetries contribute to everyday biases have largely focused on commonly-known preferences, such as handedness and footedness. Although kissing is also a frequently conducted behavior, head-turning asymmetries when kissing have received considerably less empirical attention. The characteristic that makes kissing laterality particularly unique is that it is a method of social communication that requires two partners to comply with the same lateral turning direction. In contrast, the more commonly-studied lateral preferences tend to serve functional purposes that are conducted independently (e.g., using one leg to depend on for balance), or have social contracts that establish the direction of bias (e.g., a right-handed handshake). By studying the laterality of kissing, we can explore how individuals typically engage in this form of nonverbal communication and investigate its parallels to other everyday motor asymmetries.

\section{Materials and Method}

The turning direction of 230 kissing couples were coded from 23 videos on the video-sharing website, YouTube. Videos were found by searching the original First Kiss film by Tatia Pilieva, and the remaining videos were provided by YouTube's search results which featured other First Kiss videos (see "Appendix" for list of videos). The initiation of the kiss was the point of interest for coding the turning direction; therefore, longer kisses that varied in turning direction were only counted once, and that was at the first point of lip contact. The variables coded were the turning direction of the kiss (left $=-1$, center $=0$, right $=1$, and 2 =ambiguous) and kissing partners (man-woman, man-man, woman-woman; see Table 1 for kissing partner information). Four images were classified as ambiguous due to occluded views of the couple's faces due to the camera angle, and were excluded from

Table 1 Frequencies for gender of kissing dyads and turning direction observed from sample of first kiss videos

\begin{tabular}{lrl}
\hline Kissing group & $N$ & Left/central/right turns \\
\hline Man-Woman & 187 & $86 / 2 / 99$ \\
Man-Man & 17 & $11 / 0 / 6$ \\
Woman-Woman & 22 & $12 / 0 / 10$ \\
Total & 226 & $109 / 2 / 115$ \\
\hline
\end{tabular}


analysis; this resulted in a total of 226 kissing couples in our sample. Coding was completed on all First Kiss videos that were presently available.

\section{Inter-coder Reliability}

Turning direction in all the videos was coded independently by the primary researcher and a second coder who was blind to the hypotheses of the study. Screen captures from each video during the initiation of the kiss were compiled to ensure that coders' responses were toward the same couple and to identify any duplicate footage of the couples, as they were often featured more than once per video. Coding disagreements were only found when one coder perceived a lateral turning direction and the other could not identify the direction (i.e., an ambiguous turn); ambiguous kissing directions were excluded from data analysis. A Cohen's Kappa was conducted and revealed that turning direction was in high agreement between coders, $\mathrm{K}=.96, p<.001,95 \%$ CI $[.944, .983]$.

\section{Results}

From the 226 couples observed in the First Kiss videos, almost a 1:1 ratio was exhibited between left $(48.2 \%)$ and right $(50.9 \%)$ turns; the remaining .9\% of kisses displayed no bias (i.e., a central kiss), and were excluded from the sample prior to analysis. The proportion of observed lateral turning biases did not significantly differ from the expected $50 \%, \chi^{2}$ $(1)=0.04, p=.85$, which indicates the absence of a unidirectional turning bias displayed by strangers sharing a first kiss.

To examine whether the non-significant results were because of a lack of statistical power, a post hoc power analysis for a Chi square test was conducted using the software GPower (Faul et al. 2007). To compute achieved power, the following input parameters were set: alpha's error of probability to .05 , total sample size to 226 , degrees of freedom to 1 , and the effect size used to detect a large effect $(w=.5)$. The post hoc power reported is 1.00 , which demonstrates that a sufficient sample size was examined in order to find a potential effect (O'Keefe 2007). Our negative findings therefore cannot be interpreted to be due to our sample size of 226 observations.

In light of our findings, we compared the proportion of lateral turning biases observed from the original observational study by Güntürkün (2003) to examine if the direction of turning bias was significantly different from that of the current study; to reiterate, from the 124 couples observed in the previous study, $65 \%$ were directed to the right, and $35 \%$ were to the left (see Fig. 2 for comparisons). The results from a Chi square statistical test revealed that the right-turn bias exhibited from the previous study is higher that of the current study, $\chi^{2}(1)=5.63, p=.017$; more specifically, the context of a presumed romantic kiss exhibited by couples in the previous study portrayed significantly more right-head turns than strangers conducting a first kiss.

\section{Discussion}

The current study examined the kissing orientation of strangers in videos from the First Kiss social media trend. When leaning forward to kiss a stranger, we predicted that couples would display a rightward head-turning bias which is consistent to the turning 
Fig. 2 Proportion of right and left head-turning biases between Güntürkün's (2003) original kissing bias study and current study

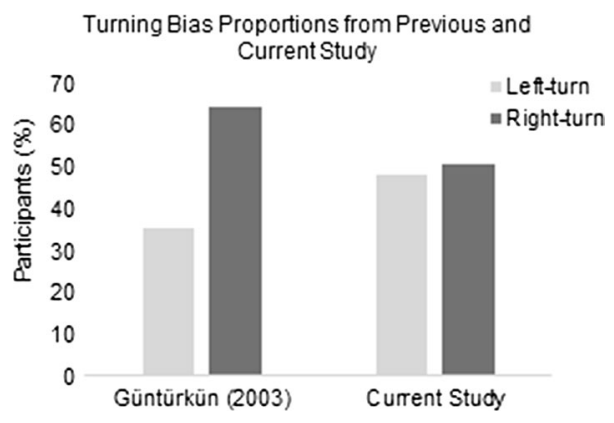

direction of lip-kissing from romantic couples in Western cultures (Barrett et al. 2006; Güntürkün 2003; Ocklenburg and Güntürkün 2009; Shaki 2013; van der Kamp and Canal-Bruland 2011). This prediction was guided by the previous literature which posited that the right head-turn preference exhibited from kissing behavior results from a corresponding motor bias observed from 38-weeks of gestation (Konishi et al. 1986; Ververs et al. 1994). Our results revealed that no significant directional bias was found, as the amount of left and right turns were nearly equal. The findings from our novel scenario contrast that of previous studies examining adult kissing, thus disputing the predominant theory that head-turning from kissing behavior results from an innately determined motor preference.

An important aspect to note is that the lack of bias observed during strangers kissing was revealed using kissing criteria parallel to the previous research. Although our observations were from existing media rather than naturalistic environments, the only difference that is relevant to turning bias is the situational context. Therefore, we can justly conclude that the attenuated turning asymmetry for strangers kissing depended on the type of kiss.

In general, the purpose and motivation in delivering a kiss to a romantic partner differs in comparison to a stranger. The romantic kiss is defined by its relational and sexual motives (Moore et al. 2017), and its quality and frequency in established relationships is found to be positively associated with partner satisfaction (Wlodarski and Dunbar 2015). The significance of giving a physically pleasurable kiss is perhaps consciously understood as evident from the volume of online content dedicated to informing viewers how to give the "ultimate" kiss. Interestingly, the intimacy involved in kissing is demonstrated from qualitative interviews with two categories of sex workers: street walkers and escorts. While the primary service offered by street walkers is pleasurable in the physical sense, escorts also offer emotional pleasure via affection (e.g., company, conversation) to deliver a "girlfriend experience" (Carbonero and Gómez Garrido 2018). Although kissing is a physical act, street walkers define kissing as a "body exclusion zone" (Sanders 2005) and refuse to kiss clients under the notion that kisses are deemed "too intimate" (Brewis and Linstead 2000); alternatively, kissing is a fundamental service provided by escorts (Carbonero and Gómez Garrido 2018). Overall, romantic kissing is incomparably motivated by emotions that express intimacy, physical pleasure, and passion.

Conceivably, kisses between strangers from the First Kiss videos did not share these same elements. Although participation was consensual, the motivation to deliver or the expectation to receive a passionate kiss was predictably lower than that of a romantic one, and likely an uncomfortable experience considering that most kisses were filmed in 
North American cities that do not conduct even social forms of kissing. By acknowledging the utility and motivational differences between romantic and non-romantic conventions of kissing, we speculate that the lack of bias resulted from the decreased initiative to deliver a pleasurable kiss. With respect to other behavioral asymmetries (e.g., seating biases, posing biases), the type of affective motivation can influence the directionality of bias.

Turning behaviors of individuals entering a symmetrical theatre are found to depend on the purpose and motivation of the presentation. When examining real-world seating biases of movie-goers, individuals display a right-side bias (Harms et al. 2014); however, when participants are told they are negatively motivated to view the film, the right-directional bias disappears (Okubo 2010). Lateral head-turning in picture posing similarly differs by the context in which it is framed. Participants asked by Nicholls et al. (1999) to portray as much emotion as possible offered the left cheek (i.e., a right head-turn), but when instructed to withhold emotion, a left-turn, right-cheek bias was presented. Curiously, the theory grounding these turning biases is not guided by a congenital mechanism, but is speculated to arise from functional differences between cerebral hemispheres. Given the attenuated bias when presenting a novel kissing context in the current study, we question whether kissing laterality is similarly guided by hemispheric asymmetries rather than from a congenital mechanism. The following section provides a speculative account for the rightward direction of romantic kissing and attenuated bias for strangers kissing.

\section{Hemispheric Lateralization of Emotion Processing}

The approach-withdrawal model of emotional lateralization operates under the premise that functional differences between cerebral hemispheres guide the direction of behavioral asymmetries. According to this model of emotional lateralization, emotions such as happiness and anger are associated with approach-motivation, best characterized as goaloriented, reward-driven behavior (Davidson and Irwin 1999). Approach-oriented behaviors are neurologically found to correspond with left-hemisphere activation; when positive affect is promoted, a relative increase in the left prefrontal cortex is exhibited (HarmonJones and Allen 1998; Tomarken et al. 1992), thus increasing attention to the contralateral right visual-field. Alternatively, the withdrawal system is specialized for most negative emotions (i.e., disgust, fear) to which the behavior is goal-aversive. Contrasting approachmotivation, the withdrawal system is associated with right-hemisphere specialization (Davidson et al. 1990), thus facilitating an opposite leftward bias.

Rightward biases arising from approach-related contexts are demonstrated from various visuospatial behaviors. During line-bisection tasks, a common measure of assessing lateral visuospatial bias (Bowers and Heilman 1980; Jewell and McCourt 2000), individuals provided with a positive outcome (approach-motivation) bisect lines significantly farther to the right than individuals assigned to a negative outcome (withdrawal-motivation) group (Friedman and Förster 2005; Nash et al. 2010). Arising from this model of emotional laterality, we consider whether romantic kisses correspond with approach-motivated behavior, thus facilitating more right-turns from the left hemisphere's goal-oriented emotional function. Strangers kissing creates a unique condition, in which individuals have assumingly lower motivation than romantic kissers to provide a quality kiss, and this condition perhaps shifts the direction of bias due to withdrawal or decreases approach motivation. Further reasoning comes from the observation that couples from the First Kiss videos were 
predominantly from North American cities that do not embody social norms of kissing, also contributing to higher avoidance-related behavior.

\section{Limitations}

Due to the ethical constraints of asking unacquainted participants to kiss, our only conceivable option to examine the kissing laterality of strangers was the circumstantial occurrence of the First Kiss video phenomenon. However, we note several limitations that stem from the nature of employing a content analysis.

Obtaining qualitative information from participants regarding their feelings towards their kissing partner and the shared kiss would have been fruitful for the present study. For example, asking participants to rate their feelings prior to meeting their kissing partner, their physical attraction toward the stranger, and their quality of the kiss could illuminate approach versus withdrawal emotions linked to the kiss's turning direction. We could possibly make these assumptions from the duration that couples kissed, though each video pans from the initiation of each kiss to feature other couples, thereby obscuring the kiss's duration. Personal questions of the participants could also be collected such as what motivated them to volunteer to kiss a stranger, their relationship status at the time of the video, or number of kissing partners, which could indicate the comfort of kissing a stranger. For instance, those in relationships may be less reluctant to engage in the kiss than those who are not, even if the kiss were consensual. Whether participants conduct kissing as a social gesture could also be of interest. Specifically, those whose cultural customs include social kissing may have established a head-turning direction when kissing non-romantic partners. This is supported from research by Chapelain et al. (2015) which found that head-turns for social cheek kissing varied by regions in France, but that the direction was almost unanimously followed by members from each region.

Other aspects regarding demographic or participant information from the kissers are also worth considering. Cultural driving customs (i.e., driving on the left or right side of the road) could theoretically facilitate a disparate turning bias, as Scharine and McBeath (2002) found an attenuated right-turn bias in a T-maze task between British and American participants. However, the right-turn bias is observed from studies conducted in geographic locations with both right-lane driving patterns (e.g., Belfast; Barrett et al. 2006) and left ones (e.g., United States, Germany; Güntürkün 2003). Lastly, motor preferences (e.g., handedness, footedness) could be recorded, as this information is important when investigating lateralized behaviors. To note, however, is that the extant literature exploring the joint pattern between head-turning when kissing and other motor biases are mixed. Ocklenburg and Güntürkün (2009) reported a significant relationship of turning bias for doll kisses to handedness and footedness; however, van der Kamp and Canal-Bruland 2011, Barrett et al. (2006), and Shaki (2013) found no correlations between turning preference respective to handedness, footedness, or eyedness. Overall, the novelty of our kissing context was at the expense of addressing the preceding information. However, the extant literature observing authentic kissing behavior (e.g., Güntürkün 2003) also lacks descriptive information of the kissers. Our primary focus of this study was, therefore, to specifically compare the direction of lateral turning bias.

Akin to the limitation of the absence of demographic data, the present study would benefit from information regarding the creation of the videos. Specifically, the videos' production and editing could inform the degree of artificiality of the kisses. When 
filming, for example, the orientation of the camera and the instructions from the director to the kissers could have subsequently influenced the direction of head-turns. When editing, the videos, in theory, could have been systematically chosen to feature both left and right turns to make the footage more varied. We also do not know if the cameras produced reversal imaging, as the footage could have portrayed mirror images of the kissing couples. This would only influence the results if this was the functionality for some, but not all cameras that filmed the First Kiss videos.

A defense for the limitation regarding video editing and production stems from the context of which the First Kiss videos achieved their popularity. The original First Kiss video by Tatia Pllieva was initially a viral media phenomenon; within the first month, the video had 77.8 million views on YouTube and was shared 1,392,296 times on Facebook (Marshall 2014). However, once viewers learned that this video was branded content of Wren, a considerably unknown retail brand, and that the kissers were not ordinary people, but model friends of the director, the attention swiftly turned negative (Leiderman 2014). Arising from this feeling of deceit, First Kiss videos began accumulating to provide a rendition of a true "first kiss". Although we still cannot be certain of the authenticity of the kisses or video editing, we have more reason to assume that the videos were edited less than other ones because of the backlash of authenticity from the original First Kiss video.

\section{Concluding Remarks}

In conclusion, the present study provides insight on kissing behaviors from a novel perspective: a first kiss shared between strangers. Our research demonstrates that the right-turn bias reliably exhibited between Western couples is not facilitated in firsttime kissing encounters between non-romantic partners, as no significant directional bias was found. This directionality may be guided by hemispheric differences associated with motivation, as choosing strangers to kiss from the First Kiss videos from our study and the naturalistic paradigms from previous studies may respectively activate approach and withdrawal motivations. Future empirical contributions should focus on the mechanisms guiding this kissing asymmetry, particularly regarding other conventions of kissing.

Acknowledgements This research was supported by a grant from the Natural Sciences and Engineering Research Council of Canada awarded to L. J. Elias.

\section{Compliance with Ethical Standards}

Conflict of interest The authors declare that they have no conflict of interest.

Ethical Approval Our data was sampled from publicly available media rather than participant generated data; therefore, informed consent was not possible. The University of Saskatchewan Research Ethics Board indicates that publicly available content is not subject to review (go to http://research.usask.ca/for-researchers/ policies-and-procedures.php and select to Human Ethics Policies, University of Saskatchewan Policies and Procedures for Ethics in Human Research, and see Section 3.2a).

Open Access This article is distributed under the terms of the Creative Commons Attribution 4.0 International License (http://creativecommons.org/licenses/by/4.0/), which permits unrestricted use, distribution, 
and reproduction in any medium, provided you give appropriate credit to the original author(s) and the source, provide a link to the Creative Commons license, and indicate if changes were made.

\section{Appendix}

\section{First Kiss Videos}

\begin{tabular}{|c|c|c|}
\hline Location of video & Number of couples & Video title \\
\hline Berkeley, USA & 12 & First Kiss at Berkeley \\
\hline Boston, USA & 16 & First Kiss: Emerson College Edition \\
\hline Coral Gables, USA & 3 & First Kiss AUC Edition \\
\hline Durham, USA & 12 & Duke University's 'First Kiss' \\
\hline Evanston, USA & 14 & Northwestern's First Kiss \\
\hline Fresno, USA & 9 & Edison High First Kiss \\
\hline Los Angelos, USA & 10 & WREN Presents: First Kiss \\
\hline New York, USA & 16 & First Kiss NYC \\
\hline Oakville, USA & 9 & First Kiss Students Edition: Sheridan College \\
\hline Philadelphia, USA & 7 & First Kiss: UPenn Edition \\
\hline Santa Barbara, USA & 8 & First Kiss - Real Life Edition \\
\hline Tallahassee, USA & 5 & First Kiss: HBCU Edition \\
\hline Winston-Salem, USA & 8 & First Kiss: Wake Forest Edition \\
\hline Saskatoon, CA & 10 & First Kiss: YXE \\
\hline Bristol, UK & 9 & Kissing Strangers: Bristol University \\
\hline London, UK & 9 & First Kiss: The Dating Experiment \\
\hline Caserta, IT & 6 & IL PRIMO BACIO (FIRST KISS Italy) \\
\hline Torino, IT & 9 & First Kiss: Real Life Edition Italia \\
\hline Utrecht, NL & 5 & First Kiss: Utrecht Edition \\
\hline Cologne, DE & 32 & First Kiss Cologne \\
\hline Moscow, RU & 5 & First Kiss in Moscow \\
\hline Perm, RU & 6 & Поцелуй с незнакомцем PERMM \\
\hline Yekaterinburg, RU & 10 & Поцелуй незнакомца (A kiss of a stranger) \\
\hline
\end{tabular}

\section{References}

Ardila, A., Ardila, O., Bryden, M. P., Ostrosky, F., Rosselli, M., \& Steenhuis, R. (1989). Effects of cultural background and education on handedness. Neuropsychologia, 27(6), 893-897. https://doi. org/10.1016/0028-3932(89)90013-4.

Barrett, D., Greenwood, J. G., \& McCullagh, J. F. (2006). Kissing laterality and handedness. Laterality, 11(6), 573-579. https://doi.org/10.1080/13576500600886614.

Bowers, D., \& Heilman, K. M. (1980). Pseudoneglect: Effects of hemispace on a tactile line bisection task. Neuropsychologia, 18(4), 491-498. https://doi.org/10.1016/0028-3932(80)90151-7.

Brewis, J., \& Linstead, S. (2000). 'The worst thing is the screwing'(1): Consumption and the management of identity in sex work. Gender, Work \& Organization, 7(2), 84-97. https://doi.org/10.1111/14680432.00096. 
Carbonero, M. A., \& Gómez Garrido, M. (2018). Being like your girlfriend: Authenticity and the shifting borders of intimacy in sex work. Sociology, 52(2), 384-399. https://doi.org/10.1177/0038038516 688609.

Chapelain, A., Pimbert, P., Aube, L., Perrocheau, O., Debunne, G., Bellido, A., et al. (2015). Can population-level laterality stem from social pressures? Evidence from cheek kissing in humans. PLoS ONE, 10(8), e0124477. https://doi.org/10.1371/journal.pone.0124477.

Davidson, R. J., Ekman, P., Saron, C. D., Senulis, J. A., \& Friesen, W. V. (1990). Approach-withdrawal and cerebral asymmetry: Emotional expression and brain physiology. I. Journal of Personality and Social Psychology, 58(2), 330-341. https://doi.org/10.1037/0022-3514.58.2.330.

Davidson, R. J., \& Irwin, W. (1999). The functional neuroanatomy of emotion and affective style. Trends in Cognitive Sciences, 3(1), 11-21. https://doi.org/10.1016/S1364-6613(98)01265-0.

Dragovic, M. (2004). Towards an improved measure of the Edinburgh Handedness Inventory: A one-factor congeneric measurement model using confirmatory factor analysis. Laterality, 9, 411-419. https://doi. org/10.1080/13576500342000248.

Elias, L. J., Bryden, M. P., \& Bulman-Fleming, M. B. (1998). Footedness is a better predictor than is handedness of emotional lateralization. Neuropsychologia, 36(1), 37-43. https://doi.org/10.1016/S0028 -3932(97)00107-3.

Faul, F., Erdfelder, E., Lang, A.-G., \& Buchner, A. (2007). G*Power 3: A flexible statistical power analysis program for the social, behavioral, and biomedical sciences. Behavior Research Methods, 39, 175-191. https://doi.org/10.3758/BF03193146.

Friedman, R. S., \& Förster, J. (2005). Effects of motivational cues on perceptual asymmetry: Implications for creativity and analytical problem solving. Journal of Personality and Social Psychology, 88(2), 263. https://doi.org/10.1037/0022-3514.88.2.263.

Gilbert, A., \& Wysocki, C. (1992). Hand preference and age in the United States. Neuropsychologia, 30, 601-608. https://doi.org/10.1016/0028-3932(92)90065-T.

Güntürkün, O. (2003). Human behaviour: Adult persistence of head-turning asymmetry. Nature, 421, 711. https://doi.org/10.1038/421711a.

Harmon-Jones, E., \& Allen, J. J. (1998). Anger and frontal brain activity: EEG asymmetry consistent with approach motivation despite negative affective valence. Journal of Personality and Social Psychology, 74(5), 1310-1316. https://doi.org/10.1037/0022-3514.74.5.1310.

Harms, V., Reese, M., \& Elias, L. J. (2014). Lateral bias in theatre-seat choice. Laterality: Asymmetries of Body, Brain and Cognition, 19(1), 1-11. https://doi.org/10.1080/1357650x.2012.746349.

Hopkins, B., Lems, W., Janssen, B., \& Butterworth, G. (1987). Postural and motor asymmetries in newlyborns. Human Neurobiology, 6, 153-156.

Jewell, G., \& McCourt, M. E. (2000). Pseudoneglect: A review and meta-analysis of performance factors in line bisection tasks. Neuropsychologia, 38(1), 93-110. https://doi.org/10.1016/S0028-3932(99)00045 $-7$.

Konishi, Y., Mikawa, H., \& Suzuki, J. (1986). Asymmetrical head-turning of preterm infants: Some effects on later postural and functional lateralities. Developmental Medicine and Child Neurology, 28(4), 450457. https://doi.org/10.1111/j.1469-8749.1986.tb14282.x.

Kumar, S., Misra, I., Suman, S., Suar, D., \& Mandal, M. K. (2010). Interrelationship of limb dominance and sensory function across age. International Journal of Neuroscience, 120(2), 110-114. https://doi. org/10.3109/00207450903337168.

Leiderman, L. (2014, March 30). Where Wren Studio's “First Kiss” went wrong. Retrieved June 01, 2017, from https://medium.com/@ pilotpmr/where-wren-studios-first-kiss-went-wrong-247c92e273db.

Mandal, M. K., Pandey, G., Singh, S. K., \& Asthana, H. S. (1992). Degree of asymmetry in lateral preferences: Eye, foot, ear. The Journal of Psychology, 126(2), 155-162. https://doi.org/10.1080/00223 980.1992.10543350.

Marshall, C. (2014, December 2). 'First kiss' most viewed video advertising campaign of 2014. Retrieved June 01, 2017, from http://tubularinsights.com/first-kiss-viewed-video-advertising-campaign-2014/.

Moore, E. A., Kulibert, D., \& Thompson, A. E. (2017). Is a kiss just a kiss?: Predicting variations in motives for romantic kissing. Journal of Relationships Research. https://doi.org/10.1017/jrr.2017.4.

Nash, K., Mcgregor, I., \& Inzlicht, M. (2010). Line bisection as a neural marker of approach motivation. Psychophysiology, 47(5), 979-983. https://doi.org/10.1111/j.1469-8986.2010.00999.x.

Nicholls, M. E., Clode, D., Wood, S. J., \& Wood, A. G. (1999). Laterality of expression in portraiture: Putting your best cheek forward. Proceedings of the Royal Society of London B: Biological Sciences, 266(1428), 1517-1522. https://doi.org/10.1098/rspb.1999.0809.

Ocklenburg, S., \& Güntürkün, O. (2009). Head-turning asymmetries during kissing and their association with lateral preference. Laterality, 14(1), 79-85. https://doi.org/10.1080/13576500802243689. 
O'Keefe, D. J. (2007). Brief report: post hoc power, observed power, a priori power, retrospective power, prospective power, achieved power: Sorting out appropriate uses of statistical power analyses. Communication Methods and Measures, 1(4), 291-299. https://doi.org/10.1080/19312450701641375.

Okubo, M. (2010). Right movies on the right seat: Laterality and seat choice. Applied Cognitive Psychology, 24(1), 90-99. https://doi.org/10.1002/acp.1556.

Previc, F. H. (1991). A general theory concerning the prenatal origins of cerebral lateralization in humans. Psychological Review, 98(3), 299. https://doi.org/10.1037/0033-295X.98.3.299.

Rönnqvist, L., \& Hopkins, B. (1998). Head position preference in the human newborn: A new look. Child Development, 69(1), 13-23. https://doi.org/10.1111/j.1467-8624.1998.tb06129.x.

Sanders, T. (2005). 'It's just acting': Sex workers' strategies for capitalizing on sexuality. Gender, Work \& Organization, 12(4), 319-342. https://doi.org/10.1111/j.1468-0432.2005.00276.x.

Scharine, A. A., \& McBeath, M. K. (2002). Right-handers and Americans favor turning to the right. Human Factors, 44(2), 248-256. https://doi.org/10.1518/0018720024497916.

Sedgewick, J. R., \& Elias, L. J. (2016). Family matters: Directionality of turning bias while kissing is modulated by context. Laterality: Asymmetries of Body, Brain and Cognition, 21(4-6), 662-671. https://doi. org/10.1080/1357650x.2015.1136320.

Shaki, S. (2013). What's in a kiss? Spatial experience shapes directional bias during kissing. Journal of Nonverbal Behavior, 37(1), 43-50. https://doi.org/10.1007/s10919-012-0141-x.

Tomarken, A. J., Davidson, R. J., Wheeler, R. E., \& Doss, R. C. (1992). Individual differences in anterior brain asymmetry and fundamental dimensions of emotion. Journal of Personality and Social Psychology, 62(4), 676. https://doi.org/10.1037/0022-3514.62.4.676.

van der Kamp, J., \& Canal-Bruland, R. (2011). Kissing right? On the consistency of the head-turning bias in kissing. Laterality, 16(3), 257-267. https://doi.org/10.1080/13576500903530778.

Ververs, I. A., de Vries, J. I., Van Geijn, H. P., \& Hopkins, B. (1994). Prenatal head position from 12-38 weeks. I. Developmental aspects. Early Human Development, 39(2), 83-91. https://doi. org/10.1016/0378-3782(94)90157-0.

Wlodarski, R., \& Dunbar, R. I. (2015). Are within-sex mating strategy phenotypes an evolutionary stable strategy? Human Ethology Bulletin, 30(1), 99-108.

Publisher's Note Springer Nature remains neutral with regard to jurisdictional claims in published maps and institutional affiliations. 